\title{
Unsteady MHD Pulsatile Blood Flow through Porous Medium in a Stenotic Channel with Slip at the Permeable Walls Subjected to Time Dependent Velocity (Injection/Suction)
}

\author{
Islam M. Eldesoky \\ Basic Engineering Sciences Department, Faculty of Engineering, Menoufia University, Egypt. \\ Email: eldesokyi@yahoo.com
}

\begin{abstract}
The flow through porous boundaries is of great importance both in technological as well as biophysical flows. The present paper is concerned with the study of unsteady pulsatile flow of blood through porous medium in a time dependent constricted porous channel subjected to time dependent suction/injection at the walls of the channel. The blood flow is subjected to a constant transverse magnetic field considering blood as an incompressible electrically conducting fluid. Due to the permeability of the arterial wall the no slip condition at the wall is no longer valid and one has to consider a slip condition at the channel wall. It was obliviously better and more realistic approach to obtain the flow behaviors. Perturbation analysis is used to solve the system of equations governing the flow. With a view to illustrating the applicability of the mathematical model developed here the analytic explicit expressions of axial velocity, volumetric flow rate and wall shear stress are obtained. The computed numerical results are presented graphically for different values of the physical parameters of interest to depict the variations in axial velocity, volumetric flow rate and wall shear stress.
\end{abstract}

\section{Key Words}

Pulsatile blood flow, time dependent stenosis, porous channel, slip velocity, suction/injection, MHD, porous media.

\section{Introduction}

The study of the flow through a channel with permeable walls not only possesses a theoretical appeal but also models biological and engineering systems. The major activity of the entire cardiovascular system is to supply blood to tissues under sufficient pressure gradient to exchange materials through arterial wall. This is a twoway exchange: the nutrients are carried to tissues and cells, and the fluid returns along with the wastes of cellular metabolism. Small arteries are thin-walled and consist of endothelial cells. They contain ultra-microscopic pores through which substances of various molecular sizes can penetrate inside and pass into the lumen of the arteries from the surrounding tissues. One of the most important features of the small arteries 
is the permeability of their walls. Examples of this are found in living organisms are fluid transport mechanisms among which are blood flow in the circulatory system and transpiration cooling.

Many investigators have theoretically studied the flow of blood through permeable walls. Elshehawey and Husseny [1] studied the peristaltic transport of a magneto-fluid with porous boundaries. It has been considered that the fluid entering the flow region through one plate at the same rate as it leaving through the other plate. Sinha and Misra [2] investigated the blood flow through an artery with permeable wall. Makinde and Osalusi [3] studied MHD steady flow in a two dimension channel with permeable boundaries. Steady MHD flow through a circular vertical pipe with permeable boundaries has been investigated by Elangovan and Ratchagar [4]. Makinde and Chinyoka [5] studied the unsteady MHD flow in a porous two dimension channel with the one wall impermeable and the other porous. Recently Sattar and Waheedullah [6] investigated the unsteady flow of a viscoelastic fluid through porous medium bounded by two porous plates. It is assumed that one plate is injected with certain constant velocity and that other it is sucked off with the same velocity. Xin-Hui Si et al [7] studied the asymmetric laminar flow in a porous channel with expanding or contracting walls. Homotopy analysis method HAM was employed to obtain the expressions for velocity fields.

Due to the permeability of the arterial wall, the no slip condition at the wall is no longer valid and one has to consider a slip condition at the artery wall. The slip condition plays an important role in shear skin, spurt and hysteresis effects. The boundary conditions relevant to flowing fluids are very important in predicting fluid flows in many applications. The fluids that exhibit boundary slip have important technological applications such as in polishing valves of artificial heart and internal cavities [8]. For many fluids, such as particulate fluids, the motion is still governed by the Navier-Stokes equations, but the usual no-slip condition at the boundary should be replaced by the slip condition Beavers and Joseph [9]. In this case, the use of slip boundary condition in preference to the no slip condition was due to the fact that walls allowed the fluid particles to slip. It was obliviously better and more realistic approach to obtain the flow behaviors. Many recent researches have been made in the subject of slip boundary conditions [2-4, 8, 10-12].

In human circulatory system blood flow, under normal conditions depends on the pumping action of the heart. The pumping action of the heart produces a pressure gradient throughout the arterial and venous network. Pulsatile flow occurs in many areas of engineering fluid dynamics like pressure surges in pipelines, cavitations in hydraulic systems, pumping of slurries, refrigeration systems, combustion mechanisms, de-watering devices and cardiovascular biomechanics. Considerable attention has been given to the study of the problems of pulsatile flow of fluids in channels of various cross-sections due to their increasing application in the analysis of the blood flow and in the flows of other biological fluids [13]. 
The study of pulsatile flow in a porous channel or porous pipe has recently becomes the object of scientific research because of its importance in some practical phenomena such as transpiration cooling and gaseous diffusion. Particularly, the study of pulsatile flow in a porous channel is useful in understanding the process of dialysis of blood in artificial kidney and in industrial applications in relation to heat exchange efficiency. Also, the pulsatile flow between permeable walls is important in understanding the blood flow in the circulatory system where the nutrients are supplied to tissues of various organs and waste products are removed. In 1971, Wang [14] studied the interesting problem of pulsatile flow in a porous channel bounded by rigid walls. Many researchers studied the effect of slip velocity at the permeable boundaries [2-4]. Recently, Eldesoky [8] investigated the unsteady pulsatile flow of blood through porous medium in an artery under the influence of periodic body acceleration and slip condition in the presence of magnetic field considering blood as an incompressible electrically conducting fluid.

Most of the researchers dealing with steady incompressible laminar flow with uniform injection or suction, have attempted to determine the axial pressure variation, wall shear stress on the porous walls and shapes of the velocity profiles within the tube. The unsteady suction problem was considered by Tsangaris et al. [15]. The case of periodic suction for flow through parallel plates was considered by Ramanamurthy et al. [16]. The steady flow of micropolar fluid through a circular pipe under a transverse magnetic field with constant suction/injection at the walls of the tube has been investigated by Murthy and Bahali [17]. Recently, many researchers studied the injection/suction at the permeable walls [18-20]

The study of flow of an electrically conducting fluid through a channel permeable walls not only possesses a theoretical appeal but also model many biological and engineering problems such as magnetohydrodynamics (MHD) generators, plasma studies, nuclear reactors, geothermal energy extraction, the boundary layer control in the field of aerodynamics, blood flow problems, etc. The application of Magnetohydrodynamics in physiological flow is of growing interest. The flow of blood can be controlled by applying appropriate magnetic field. Many researchers have shown that blood is an electrically conducting fluid. The Lorentz's force will act on the constituent particles of blood and this force will oppose the motion of blood and thus reduces its velocity. This decelerated blood flow may help in the treatment of certain cardiovascular diseases and in the diseases with accelerated blood circulation such as hypertension, hemorrhages etc. So, it is very essential to study the blood flow in presence of magnetic field. Many works have been done in this field by various investigators $[8,13,21-22]$.

Atherosclerosis is a disease of the cardiovascular system which involves hardening of arteries due to the deposition of plaque. Localized atherosclerotic constrictions in arteries, known as arterial stenoses, are found predominantly in the internal carotid artery which supplies blood to the brain, the coronary artery which 
supplies blood to the cardiac muscles, and the femoral artery which supplies blood to the lower limbs. Blockage of more than about $70 \%$ (by area) of the artery is considered clinically significant since it presents significant health risks for the patient [23]. Complete closure of the artery can occur if a blood-clot becomes lodged in the stenosis and this can lead to a stroke or a heart attack. In addition to this, moderate as well as severe stenoses can have long-term health consequences. First, the presence of a constriction results in head losses which can reduce the blood supply through the artery and also impose additional load on the heart. Secondly, the fluctuations in the blood flow downstream of the stenosis can damage and weaken the internal wall (intima) of the artery. It is accepted that both the wall pressure and shear stress play a role in this.

For many decades, cardiovascular disease has been one of the most severe diseases causing a large number of deaths worldwide each year, especially in developed countries. Most of the cases are associated with some form of abnormal flow of blood in stenotic arteries. In the presence of a stenosis, the normal blood flow through the artery is disturbed resulting in blood recirculation and wall shear stress oscillation near the stenosis. The heart has to increase the blood pressure to impel the blood passing through the narrowing region so as to enforce the blood circulation. If the heart works too hard and the blood cannot flow well, heart attack may occur. In order to understand the blood flow behavior in arteries so as to provide sufficient information for clinical purposes, intensive research has been carried out worldwide for both normal and stenotic arteries. Mekheimer et al. [24-26] studied the unsteady pulsatile flow through a vertical constricted annulus with heat transfer and investigated Magnetic field and Hall currents influences on blood flow through a stenotic artery. Also, studied the induced magnetic field influences on blood flow through an anisotropically tapered elastic arteries with overlapping stenosis in an annulus. The effects of Magneto-hydrodynamic and hematocrit on blood flow in an artery with multiple mild stenosis has been investigated by Verma and Parihar [27]. Nagarani and Sarojamma [28] investigated the effect of body acceleration on pulsatile flow of Casson fluid through a mild stenosed artery. Mishra and Verma [29] studied the effect of porous parameter and stenosis on the wall shear stress for the flow of blood in human body. Sinha et al. [30] studied the mathematical modeling of blood flow in porous vessel having double stenosis in the presence of an external magnetic field.

The main objective of the present paper is to study the combined effect of magnetic field and permeable walls slip velocity on the unsteady pulsatile flow of blood through porous medium in a time dependent constricted porous channel subjected to time dependent suction/injection at the walls of the channel considering blood as an incompressible electrically conducting fluid. The governing continuity and Navier-Stokes equations are solved by perturbation technique. In the following sections, the problem is formulated, analyzed and discussed. 


\section{Mathematical model}

The simulation model of the stenosed porous channel is depicted in Fig. 1. The channel walls are located at a distance $2 \mathrm{H}$ apart with reference to a Cartesian coordinate system $(\mathrm{x}, \mathrm{y})$. Let $\mathrm{x}$-axis be taken along the axis of the channel while $\mathrm{y}$-axis is the transverse coordinate normal to $\mathrm{x}$ axis. Let us consider the pulsatile flow of blood as an electrically conducting, unsteady, viscous, incompressible and Newtonian fluid in the presence of constant magnetic field of strength $B_{o}$ acting perpendicular to the channel. We assumed that the magnetic Reynolds number of the flow is taken to be small enough, so that the induced magnetic and electric field can be neglected. We consider that the wall of the channel is permeable, so that finite fluid exchange can takes place across the wall. The channel is filled with a homogeneous porous media.

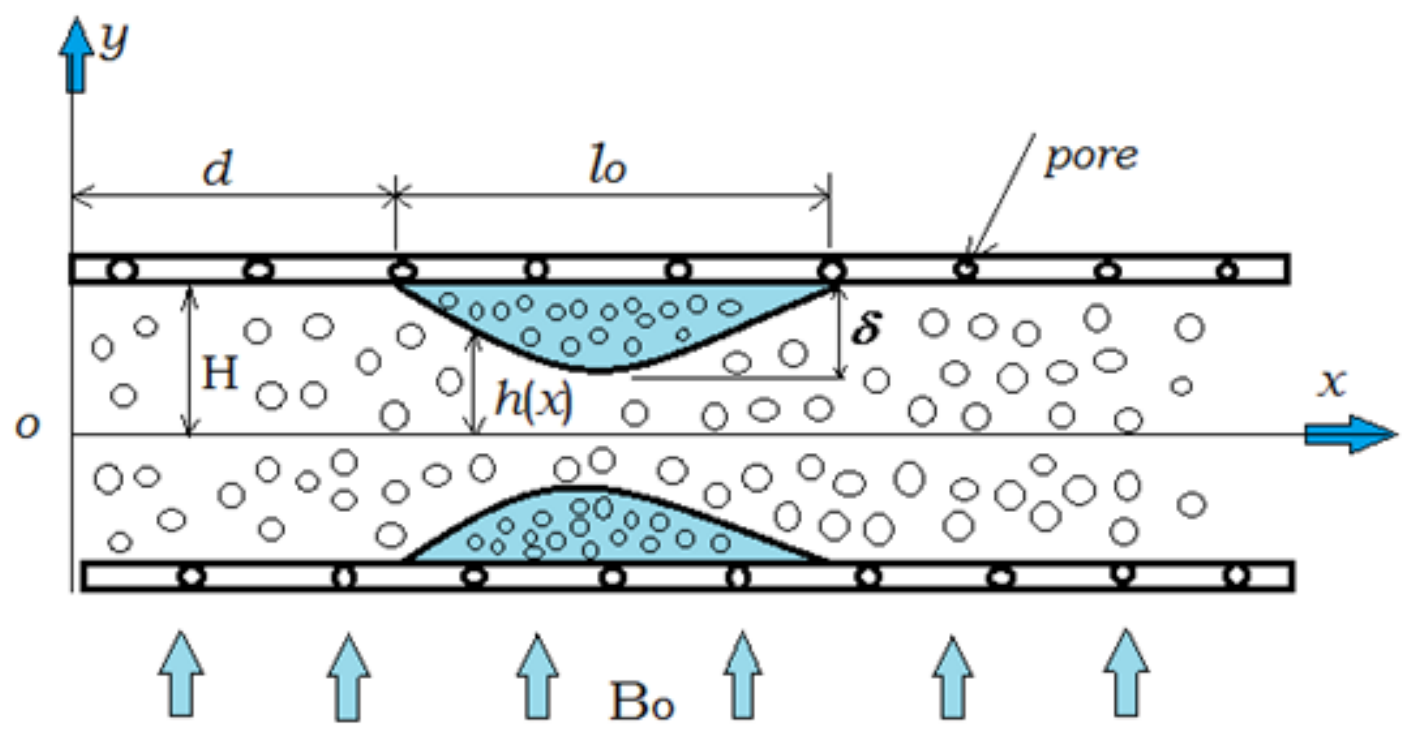

Fig. 1 Schematic representation of the model geometry

Under the above assumptions, in two dimensions, the governing equations of continuity and momentum (in $\mathrm{x}$ and $\mathrm{y}$-direction) are given in dimensional form as

$$
\begin{aligned}
& \frac{\partial u}{\partial x}+\frac{\partial v}{\partial y}=0, \\
& \frac{\partial u}{\partial t}+u \frac{\partial u}{\partial x}+v \frac{\partial u}{\partial y}=-\frac{1}{\rho} \frac{\partial p}{\partial x}+v\left(\frac{\partial^{2} u}{\partial x^{2}}+\frac{\partial^{2} u}{\partial y^{2}}\right)-\left(\frac{v}{k}\right) u-\frac{\sigma B_{o}^{2} u}{\rho}, \\
& \frac{\partial v}{\partial t}+u \frac{\partial v}{\partial x}+v \frac{\partial v}{\partial y}=-\frac{1}{\rho} \frac{\partial p}{\partial y}+v\left(\frac{\partial^{2} v}{\partial x^{2}}+\frac{\partial^{2} v}{\partial y^{2}}\right)-\left(\frac{v}{k}\right) v,
\end{aligned}
$$

where $u$ denotes the velocity component along the $\mathrm{x}$-axis, $v$ the velocity component along y-axis, t time, $v$ the kinetic viscosity, $p$ the blood pressure, $\rho$ the density of the blood, $\sigma$ the electrical conductivity and $k$ the permeability of porous medium. 
The absorption of fluid at the walls is accounted by prescribing the flow flux as Makinde and Chinyoka [5]

$$
\int_{-H}^{H} u d y=U f\left(\frac{x}{2 H}\right)\left(1+\varepsilon e^{i \omega t}\right),
$$

where $\mathrm{U}$ is the initial characteristic flow velocity (i.e. at $x=0)$ and $f(x / 2 H)$ is the flux function that describes the rate of fluid absorption through the permeable wall. We assume that the fluid is injected or sucked off through the channel walls with a time dependent velocity $\mathrm{V}$ is given by

$$
V=V_{o}\left(1+\varepsilon e^{i \omega x}\right),
$$

where $V_{o}$ be the uniform transpiration velocity (for injection $V_{o}>0$ and for suction $\left.V_{o}<0\right), \varepsilon$ is the small amplitude of oscillation and the value of $\varepsilon$ is necessarily less than unity $\varepsilon<1$. Since the fluid medium is filled with homogeneous porous material and the normal component of velocity $v=V$ is independent of $\mathrm{x}$ and $\mathrm{y}$ and thereby continuity equation (1) reduces to $\frac{\partial u}{\partial x}=0$. Therefore, the axial velocity $u$ is function of $\mathrm{y}$ and $\mathrm{t}$ only $u=u(y, t)$ and then the equation (3) simply reduces to $-\frac{\partial p}{\partial y}=\rho V_{o}\left(i \omega \varepsilon e^{i \omega t}\right)+\frac{\mu V_{o}}{k}\left(1+\varepsilon e^{i \omega t}\right)$.

Under the above assumptions, the axial momentum equation reduces to

$$
\frac{\partial u}{\partial t}+V_{o}\left(1+\varepsilon e^{i \omega t}\right) \frac{\partial u}{\partial y}=-\frac{1}{\rho} \frac{\partial p}{\partial x}+v \frac{\partial^{2} u}{\partial y^{2}}-\left(\frac{v}{k}\right) u-\frac{\sigma B_{O}^{2} u}{\rho} .
$$

Since the flow of blood is pulsatile, heart pumping, then the unsteady pressure gradient can be approximated as $[8,13]$

$-\frac{\partial p}{\partial x}=P_{S}+\varepsilon P_{O} \cos (\omega t)$,

where $P_{S}$ is the steady part of the pressure gradient and $P_{O}$ is the pulsatile amplitude. [30-32]

The geometry of the stenosis which is assumed to be symmetric is given by

$$
\begin{array}{rlrl}
h^{\prime}(x) & =H\left[1-\frac{\delta}{2}\left\{1+\cos \frac{2 \pi}{l_{o}}\left(x-d-\frac{l_{o}}{2}\right)\right\}\right]\left(1-[\cos (\omega t)-1] \varepsilon e^{-\omega \varepsilon t}\right), & d \leq x \leq d+l_{o} \\
& =H\left(1-[\cos (\omega t)-1] \varepsilon e^{-\omega \varepsilon t}\right), & & \text { otherwise }
\end{array}
$$


where $\delta$ is the maximum projection (height of the throat) of the stenosis located at $x=d+\frac{l_{o}}{2}, l_{o}$ is the stenosis length and $d$ indicates its location. Also, $h^{\prime}(x)$ is the variable height of the channel at the stenosed portion and $\varepsilon$ is the amplitude of oscillation.

Assuming that the flow of the blood to be symmetric about the centerline of the channel $(\mathrm{y}=0)$, we focus our attention to the flow in the region $0 \leq y \leq h(x)$ only.

For small permeability, the boundary condition proposed by Beavers and Joseph [9] was simplified by Saffman [33] as $\frac{d u}{d y}=\frac{\eta}{\sqrt{k_{1}}} u$ where $\eta$ is a constant depending only upon the properties of the porous material and not on its structure and $k_{1}$ is the permeability of porous material of the wall. We can replace the value of $\frac{\eta}{\sqrt{k_{1}}}$ by $\beta$. This condition is hold good in the case of unsteady flows and even if we take MHD effects into account [34]. Although the slip condition looked simple, analytically it is much more difficult than the no-slip condition, and then the boundary conditions on the wall of the porous channel are prescribed as follows:

$$
\begin{aligned}
& \frac{\partial u(y, t)}{\partial y}=0 \quad \text { at } \quad y=0 \\
& u(y, t)=\beta \frac{\partial u(y, t)}{\partial y} \quad \text { at } \quad y=h(x),
\end{aligned}
$$

Let us introduce the following dimensionless quantities:

$u^{*}=\frac{u}{V_{O}}, \quad x^{*}=\frac{x}{H}, \quad y^{*}=\frac{y}{H}, \quad t^{*}=\frac{t \omega}{2 \pi}, \quad P^{*}=\frac{P}{\rho V_{O}^{2}}, \quad \tau^{*}=\frac{\mu V_{O}}{H} \tau$

Using these dimensionless quantities in equations (6), (9-a, b) and dropping the stars, we obtain

$$
\begin{aligned}
& \frac{\alpha^{2}}{2 \pi \operatorname{Re}} \frac{\partial u}{\partial t}+\left(1+\varepsilon e^{2 \pi i t}\right) \frac{\partial u}{\partial y}=-\frac{\partial p}{\partial x}+\frac{1}{\operatorname{Re}} \frac{\partial^{2} u}{\partial y^{2}}-\left(M+\frac{1}{\lambda}\right) u, \\
& \frac{\partial u(y, t)}{\partial y}=0 \quad \text { at } \quad y=0, \\
& u(y, t)=K n \frac{\partial u(y, t)}{\partial y} \quad \text { at } \quad y=h(x) .
\end{aligned}
$$

The geometry of the stenosis in dimensionless form is given by 


$$
\begin{aligned}
h(x) & =\left(1-[\cos (2 \pi t)-1] \varepsilon e^{-2 \pi \varepsilon t}\right)\left[1-\frac{\delta}{2}\left\{1+\cos \frac{2 \pi}{l_{o}}\left(x-d-\frac{l_{o}}{2}\right)\right\}\right], d \leq x \leq d+l_{o} \\
& =1-[\cos (2 \pi t)-1] \varepsilon e^{-2 \pi \varepsilon t}, \quad \text { otherwise }
\end{aligned}
$$

where the magnetic parameter $M$, the Womersley parameter $\alpha$, the Reynolds number Re, the permeability parameter of porous medium (Darcian linear drag parameter) $\lambda$ and the Knudsen number $K n$ are defined respectively by

$M=\frac{\sigma B_{o}^{2} H}{\rho V_{o}}, \quad \alpha=H \sqrt{\frac{\rho \omega}{\mu}}, \quad \operatorname{Re}=\frac{H V_{o}}{v}, \quad \lambda=\frac{k V_{o}}{v H} \quad$ and $\quad K n=\frac{\beta}{H}$

\section{Solution of the problem}

We seek the solution of the governing equations on the form

$$
u(y, t)=u_{o}(y)+\varepsilon u_{1}(y) e^{2 \pi i t},
$$

where $\varepsilon$ is the small amplitude of oscillation and hence we can assume square and higher order terms of $\varepsilon$ to be negligibly small magnitude. Also, $u_{o}$ and $u_{1}$ are the velocity of steady state and transient state respectively. Substituting (12) into (10), (11-a) and (11-b), and comparing the coefficients of zero and first order terms of $\varepsilon$ on both sides, we obtain:

\section{(i) Steady state (zero order):}

$\frac{1}{\operatorname{Re}} \frac{d^{2} u_{o}}{d y^{2}}-\frac{d u_{o}}{d y}-\left(M+\frac{1}{\lambda}\right) u_{o}=-P_{S}$,

Subject to boundary conditions

$$
\begin{array}{llrl}
\frac{d u_{o}}{d y}=0 & \text { at } & y=0, \\
u_{o}=K n \frac{d u_{o}}{d y} & \text { at } & y & =h(x) .
\end{array}
$$

By solving (13) we can get the general solution on the form

$$
u_{o}=C_{1} e^{m_{1} y}+C_{2} e^{m_{2} y}+\frac{P_{S}}{\operatorname{Re}\left(M+\frac{1}{\lambda}\right)}
$$

where 


$$
\begin{aligned}
& m_{1}=0.5\left(\operatorname{Re}+\sqrt{\operatorname{Re}^{2}+4 \operatorname{Re}\left(M+\frac{1}{\lambda}\right)}\right), \\
& m_{2}=0.5\left(\operatorname{Re}-\sqrt{\operatorname{Re}^{2}+4 \operatorname{Re}\left(M+\frac{1}{\lambda}\right)}\right) .
\end{aligned}
$$

Substituting by the boundary conditions (14-a) and (14-b) we obtain the constants $C_{1}=\frac{m_{2}\left(\frac{P_{S}}{\operatorname{Re}\left(M+\frac{1}{\lambda}\right)}\right)}{m_{1} m_{2} K n\left(e^{m_{1} h(x)}-e^{m_{2} h(x)}\right)+m_{1} e^{m_{2} h(x)}-m_{2} e^{m_{1} h(x)}}$,

$$
C_{2}=\frac{-m_{1}\left(\frac{P_{S}}{\operatorname{Re}\left(M+\frac{1}{\lambda}\right)}\right)}{m_{1} m_{2} K n\left(e^{m_{1} h(x)}-e^{m_{2} h(x)}\right)+m_{1} e^{m_{2} h(x)}-m_{2} e^{m_{1} h(x)}}, .
$$

\section{(ii) Transient state (first order):}

$\frac{1}{\operatorname{Re}} \frac{d^{2} u_{1}}{d y^{2}}-\left(1+\varepsilon e^{2 \pi i t}\right) \frac{d u_{1}}{d y}-\left(M+\frac{1}{\lambda}+\frac{i \alpha^{2}}{\operatorname{Re}}\right) u_{1}=\frac{d u_{o}}{d y}-P_{O} \cos (2 \pi t) e^{-2 \pi i t}$,

Subject to boundary conditions

$$
\begin{array}{llrl}
\frac{d u_{1}}{d y}=0 & \text { at } & y & =0, \\
u_{1}=K n \frac{d u_{1}}{d y} & \text { at } & y & =h(x) .
\end{array}
$$

By solving (16) we can get the general solution on the form

$$
u_{1}=C_{3} e^{m_{3} y}+C_{4} e^{m_{4} y}+C_{5} e^{m_{1} y}+C_{6} e^{m_{2} y}+C_{7},
$$

where

$$
m_{3}=0.5\left(\left(\operatorname{Re}^{*}\right)+\sqrt{\left(\operatorname{Re}^{*}\right)^{2}+4 \operatorname{Re}\left(M+\frac{1}{\lambda}+\frac{i \alpha^{2}}{\operatorname{Re}}\right)}\right)
$$


$m_{4}=0.5\left(\left(\operatorname{Re}^{*}\right)-\sqrt{\left(\operatorname{Re}^{*}\right)^{2}+4 \operatorname{Re}\left(M+\frac{1}{\lambda}+\frac{i \alpha^{2}}{\operatorname{Re}}\right)}\right)$,

$\operatorname{Re}^{*}=\operatorname{Re}\left(1+\varepsilon e^{2 \pi i t}\right) \approx \operatorname{Re}$

Substituting by the boundary conditions (14-a) and (14-b) we obtain the constants $C_{3}, \ldots . ., C_{7}$ as follows:

$C_{3}=\frac{C_{31}+C_{32}+C_{7} m_{4}}{m_{3} e^{m_{4} h(x)}-m_{4} e^{m_{3} h(x)}+m_{3} m_{4} K n\left(e^{m_{3} h(x)}-e^{m_{4} h(x)}\right)}$,

$C_{31}=C_{5}\left[m_{4} e^{m_{1} h(x)}-m_{1} e^{m_{4} h(x)}+m_{1} m_{4} K n\left(e^{m_{4} h(x)}-e^{m_{1} h(x)}\right)\right]$,

$C_{32}=C_{6}\left[m_{4} e^{m_{2} h(x)}-m_{2} e^{m_{4} h(x)}+m_{2} m_{4} K n\left(e^{m_{4} h(x)}-e^{m_{2} h(x)}\right)\right]$,

$C_{4}=-\frac{m_{1} C_{5}+m_{2} C_{6}+m_{3} C_{3}}{m_{4}}$,

and

$C_{5}=\frac{\operatorname{Re} m_{1} C_{1}}{m_{1}^{2}-m_{1} \operatorname{Re}-\operatorname{Re}\left(M+\frac{1}{\lambda}+\frac{i \alpha^{2}}{\operatorname{Re}}\right)}$,

$C_{6}=\frac{\operatorname{Re} m_{2} C_{2}}{m_{2}^{2}-m_{2} \operatorname{Re}-\operatorname{Re}\left(M+\frac{1}{\lambda}+\frac{i \alpha^{2}}{\operatorname{Re}}\right)}$,

$C_{7}=\frac{P_{o} \cos (2 \pi t) e^{-2 \pi i t}}{\left(M+\frac{1}{\lambda}+\frac{i \alpha^{2}}{\mathrm{Re}}\right)}$

Substituting the expressions of $u_{o}$ and $u_{1}$ from the equations (15) and (18) in equation (12), we obtain the expression for the velocity $u(y, t)$ as

$$
\begin{aligned}
u(y, t)=C_{1} e^{m_{1} y}+C_{2} e^{m_{2} y}+\frac{P_{S}}{\operatorname{Re}\left(M+\frac{1}{\lambda}\right)} \\
\quad+\varepsilon e^{2 \pi i t}\left(C_{3} e^{m_{3} y}+C_{4} e^{m_{4} y}+C_{5} e^{m_{1} y}+C_{6} e^{m_{2} y}+C_{7}\right),
\end{aligned}
$$




\section{Rate of flow}

The volumetric flow rate $Q$ defined by

$$
Q=\int_{0}^{h(x)} u(y, t) d y .
$$

Substituting from equation (19) in equation (20) and calculating the integration yields

$$
\begin{aligned}
Q= & C_{1} \frac{e^{m_{1} h(x)}-1}{m_{1}}+C_{2} \frac{e^{m_{2} h(x)}-1}{m_{2}}+\frac{P_{S} h(x)}{\operatorname{Re}\left(M+\frac{1}{\lambda}\right)}+ \\
& \varepsilon e^{2 \pi i t}\left[C_{3} \frac{e^{m_{3} h(x)}-1}{m_{3}}+C_{4} \frac{e^{m_{4} h(x)}-1}{m_{4}}+C_{5} \frac{e^{m_{1} h(x)}-1}{m_{1}}+C_{6} \frac{e^{m_{2} h(x)}-1}{m_{2}}+C_{7} h(x)\right] .
\end{aligned}
$$

\section{Shear stress at the wall}

The non-dimensional wall shear stress is given by the relation

$$
\tau_{w}=\left[\frac{\partial u}{\partial y}\right]_{y=h(x)} .
$$

Substituting from equation (19) in equation (22), the wall shear stress can be written as

$$
\begin{aligned}
\tau_{w}=C_{1} m_{1} e^{m_{1} h(x)}+C_{2} m_{2} e^{m_{2} h(x)}+\varepsilon e^{2 \pi i t}\left[C_{3} m_{3} e^{m_{3} h(x)}+C_{4} m_{4} e^{m_{4} h(x)}\right. & +C_{5} m_{1} e^{m_{1} h(x)} \\
& \left.+C_{6} m_{2} e^{m_{2} h(x)}\right] .
\end{aligned}
$$

\section{Numerical Results and discussion}

In the present paper we concerned with studying the combined effect of magnetic field and permeable walls slip velocity on the unsteady pulsatile flow of blood through porous medium in a time dependent constricted porous channel subjected to time dependent suction/injection at the walls of the channel considering blood as an incompressible electrically conducting fluid. The analytical expressions of axial velocity, volumetric flow rate and wall shear stress derived in the preceding 
section have been computed numerically and plotted for different values of Knudsen number $K n$, the maximum projection (height of the throat) of the stenosis $\delta$, magnetic parameter $M$, Darcian linear drag parameter $\lambda$ and Reynolds number Re. We run our code for the parameters $\left(\alpha=0.5, P_{o}=7.0, l_{o}=10.0, \quad t=1.0, \quad P_{s}=10.0\right.$, and $\varepsilon=0.01)$ related to a realistic physical problem similar to the ones used by other authors [3,5,31]. The profiles of axial velocity versus dimensionless transverse $y$ coordinate at the throat of the stenosis for various physical parameters are shown in Figs. 2-5.

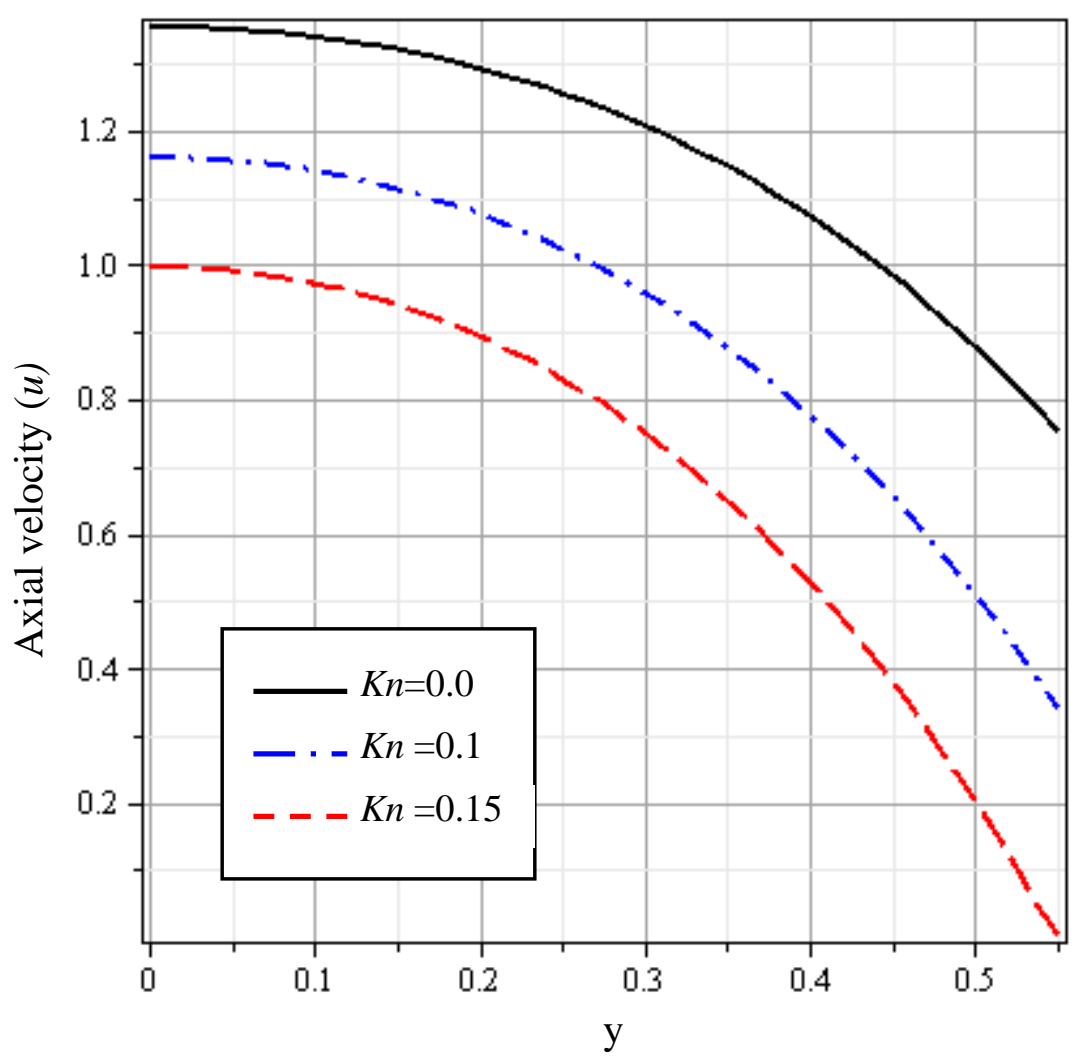

Fig. 2 Axial velocity profiles versus $y$ at the throat of the stenosis for different values of Knudsen number $K n$ when $\operatorname{Re}=1.0, M=2.0, \quad \delta=0.25$, and $\lambda=0.30$

Fig. 2 depicts that the parabolic axial velocity of blood has a maximum value at the centerline of the channel and minimum value at the walls. Also, it is clear from this figure that the axial velocity of blood decreases with increasing of Knudsen number $K n$ means that, the flowing fluid is slowed down in axial direction. 


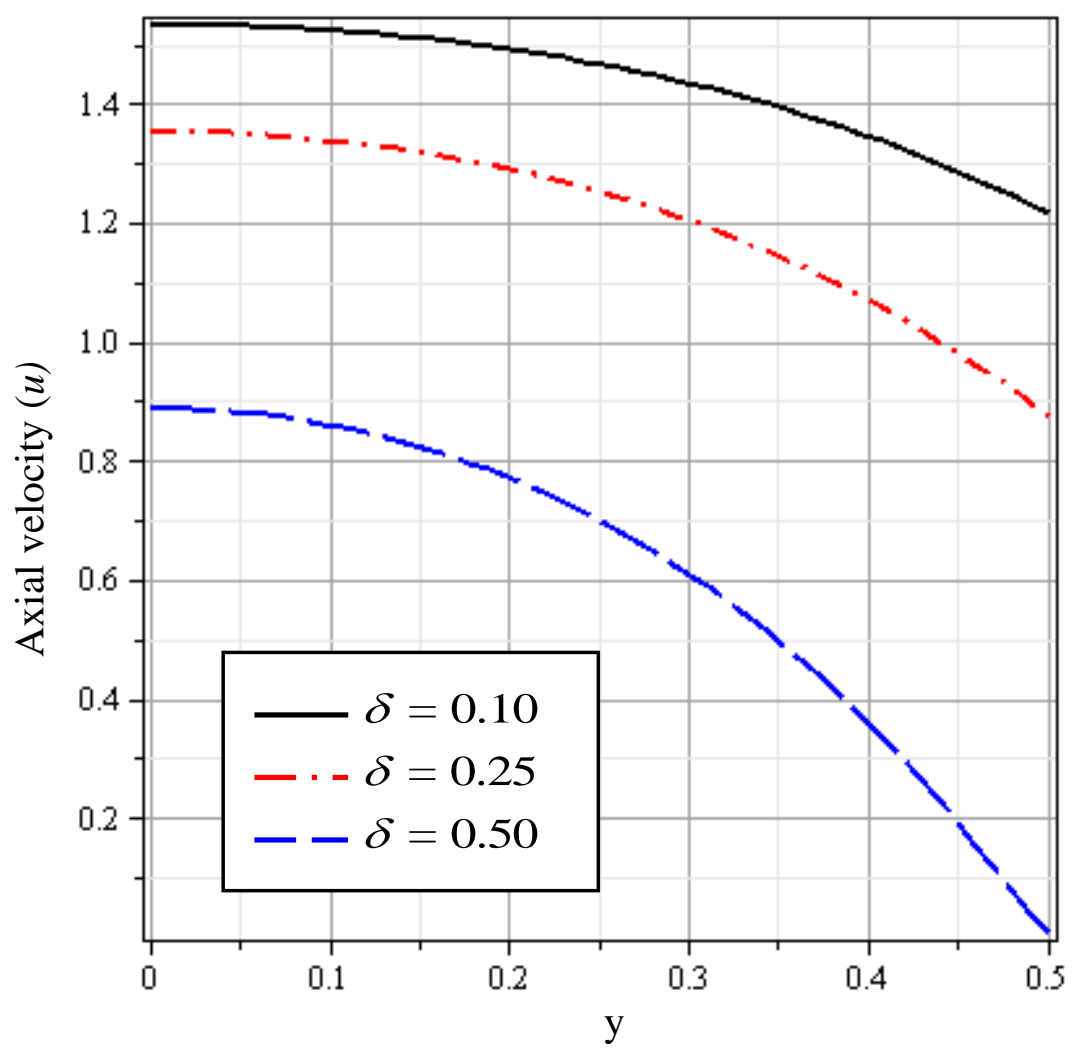

Fig. 3 Axial velocity profiles versus y at different depths of the stenosis $\delta$ when $\operatorname{Re}=1.0, \quad M=2.0, \quad K n=0.1$, and $\lambda=0.30$

Fig. 3 represents the pulsating axial velocity profiles at different locations of the channel constriction as well as different depths. It is clear that from this figure that the axial velocity strongly decreases near the walls of the channel as well as in the centerline of the channel with the increasing effects of constriction height. The effect of magnetic parameter $M$ on the pulsating axial velocity $u$ is presented in Fig. 4. It is observed that the axial velocity decreases with increasing the magnetic parameter $M$. It indicates that the blood velocity can be reduced by applying suitable magnetic field strength. Thus the reduction in blood velocity can be used for surgical patient during surgery. Fig. 5 shows that the pulsating axial velocity of the blood increases with increasing the values of Darcian linear drag parameter $\lambda$ where increasing the values of $\lambda$ corresponds to a rise in permeability and so less resistance to flow. 


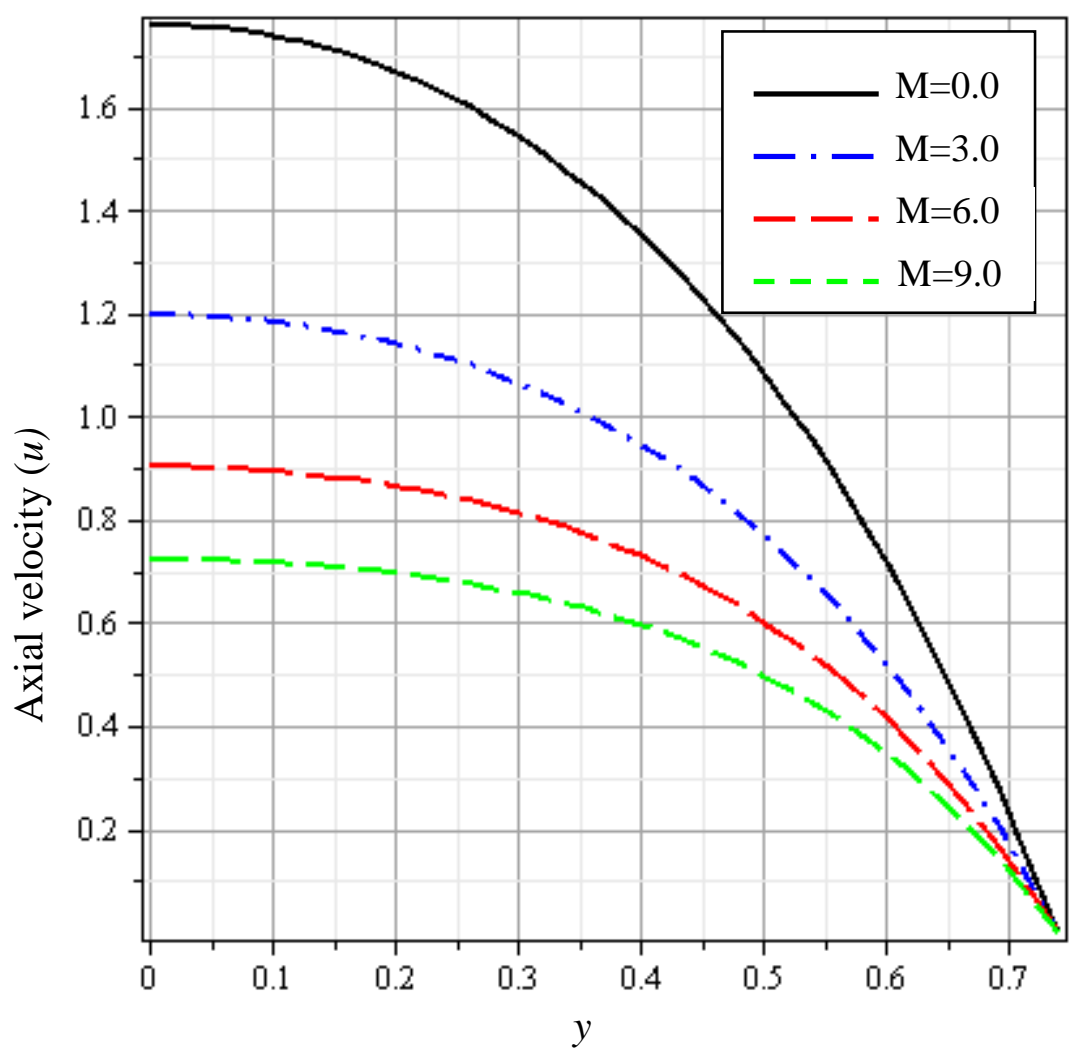

Fig. 4 Axial velocity profiles versus $y$ at the throat of the stenosis for different values of magnetic parameter $M$ when

$$
\operatorname{Re}=1.0, K n=0.1, \quad \delta=0.25 \text {, and } \lambda=0.30
$$

It is very important to note that, an increase in the positive value of flow Reynolds number $(\mathrm{Re})$ represents an increase in the fluid injection while an increase in the negative value of Reynolds number represents an increase in the fluid suction. From Fig. 6, it is observed that the pulsating axial velocity decreases with increasing the Reynolds number. It means that, at strong injection it is observed that the axial velocity profiles are concave upwards with decreasing curvature as Re increases. Moreover, for very high values of $\mathrm{Re}$, the graph is almost rectilinear.

The variations of volumetric flow rate of the blood along the dimensionless axial $x$-coordinate of the channel for various physical parameters are shown in Figs. 7-11. It is observed from Fig. 7 that the flow rate has a maximum value at the ends of the stenosis while has the minimum value at the throat of the stenosis. Moreover, the flow rate decreases with increasing the slip parameter (Knudsen number Kn). From Figs. $8,9,11$, it is seen that the volumetric flow rate decreases with increasing the height of the stenosis $\delta$, the magnetic parameter $M$ and the Reynolds number Re while the volumetric flow rate increases with increasing the Darcian linear drag parameter $\lambda$ as shown in Fig. 10. 


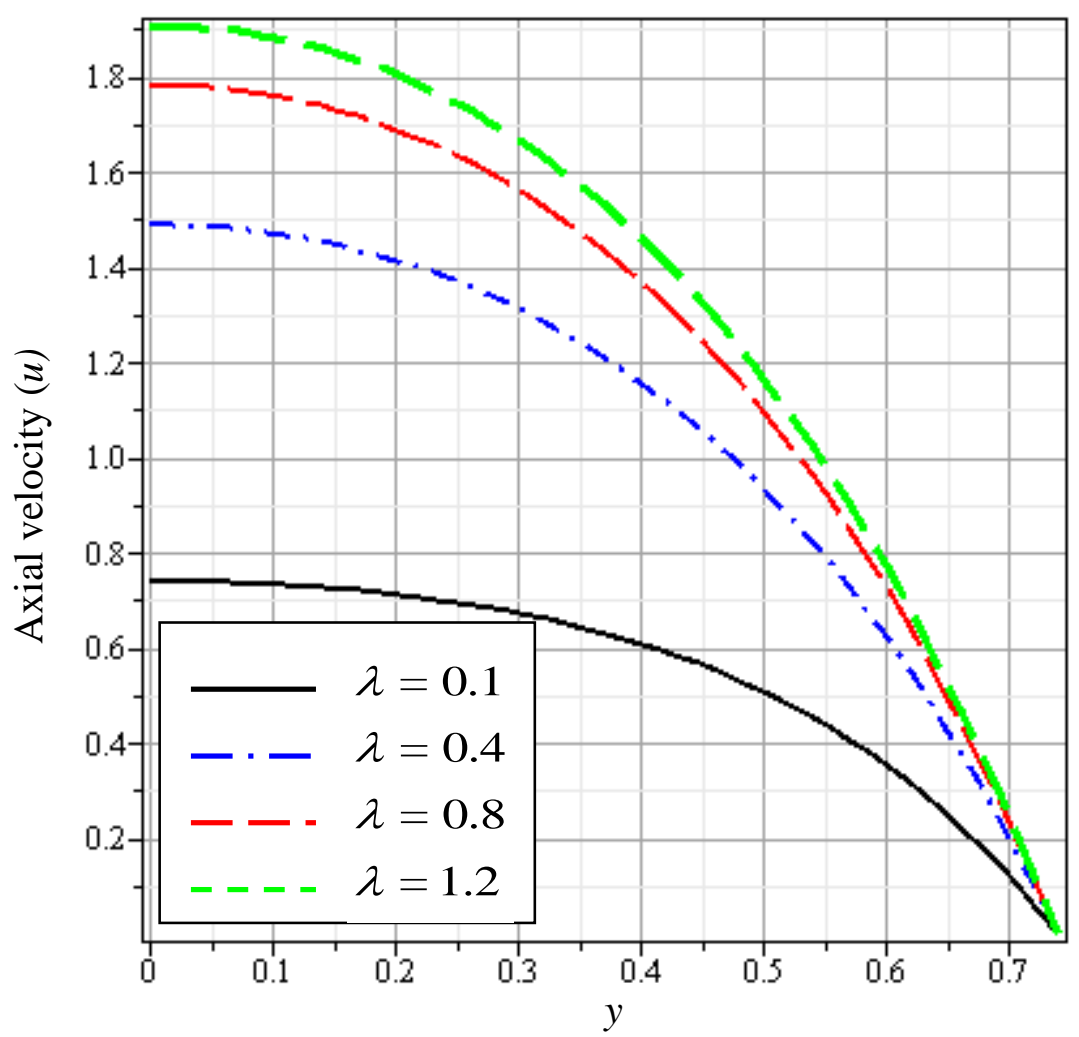

Fig. 5 Axial velocity profiles versus $y$ at the throat of the stenosis for different values of Darcian linear drag parameter $\lambda$ when $\operatorname{Re}=1.0, K n=0.1, \quad \delta=0.25$, and $\lambda=0.30$

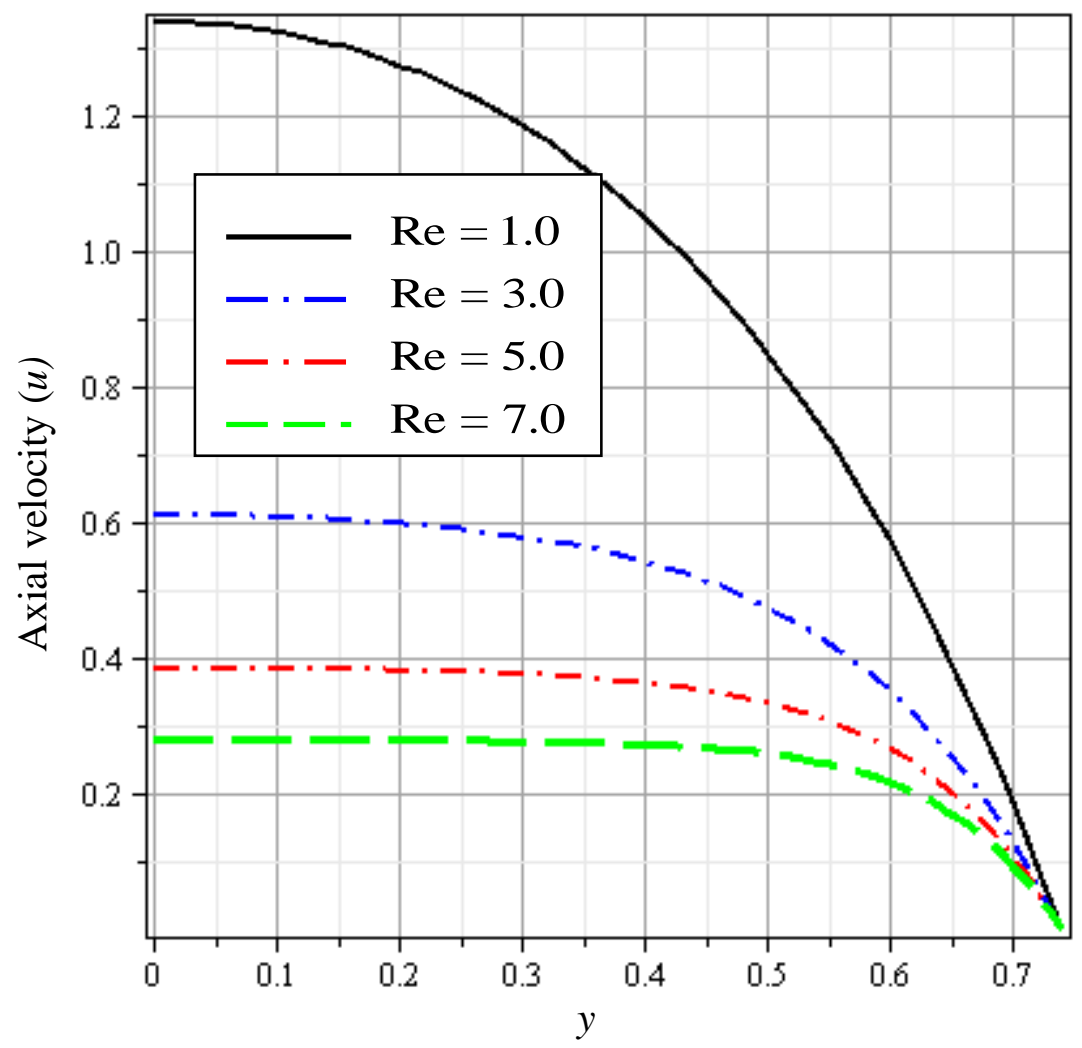

Fig. 6 Axial velocity profiles versus y at the throat of the stenosis for different values of Reynolds number Re when $M=2.0, K n=0.1, \quad \delta=0.25$, and $\lambda=0.30$ 


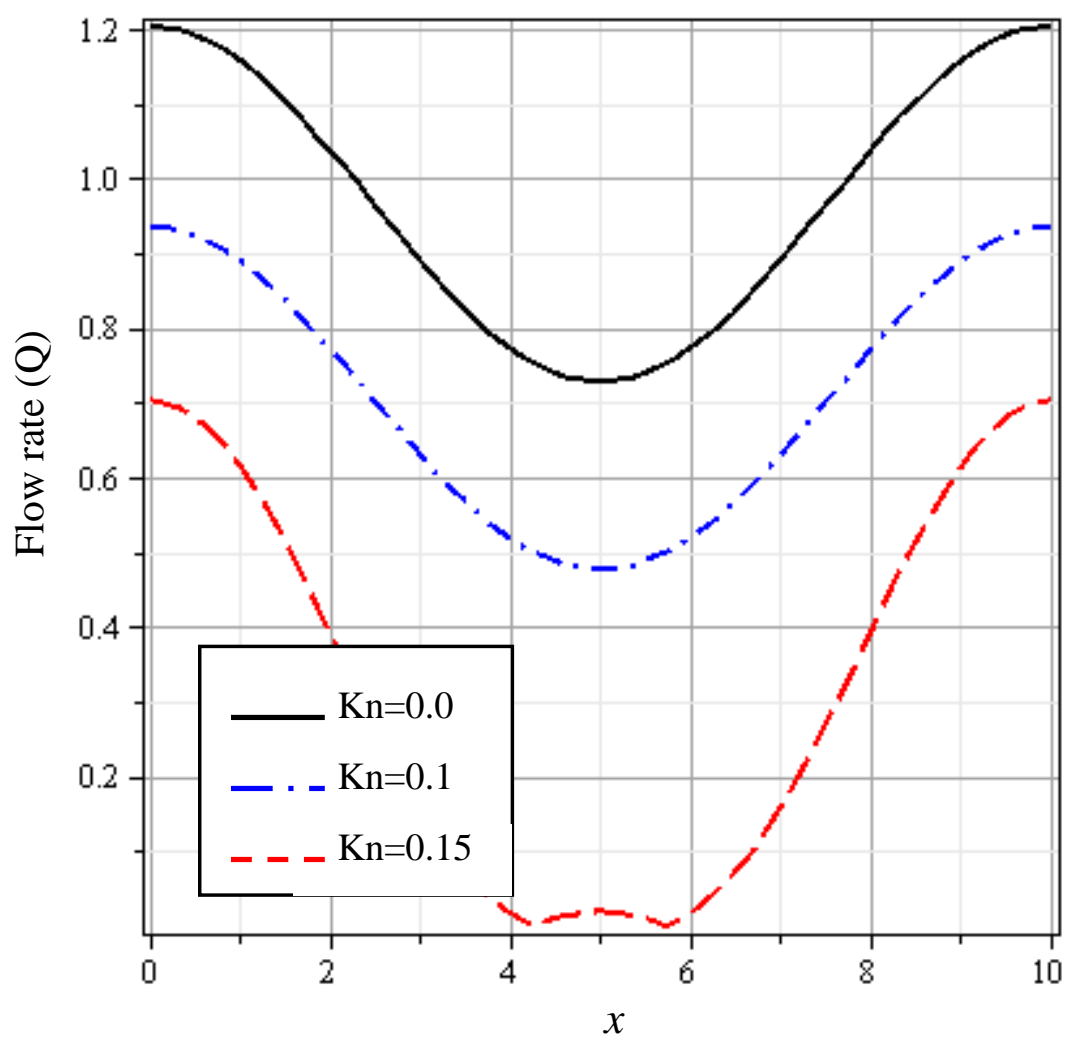

Fig.7 Variation of volumetric flow rate for ditterent values of Knudsen number $\mathrm{Kn}$ when $\operatorname{Re}=1.0, M=2.0, \delta=0.25$, and $\lambda=0.30$

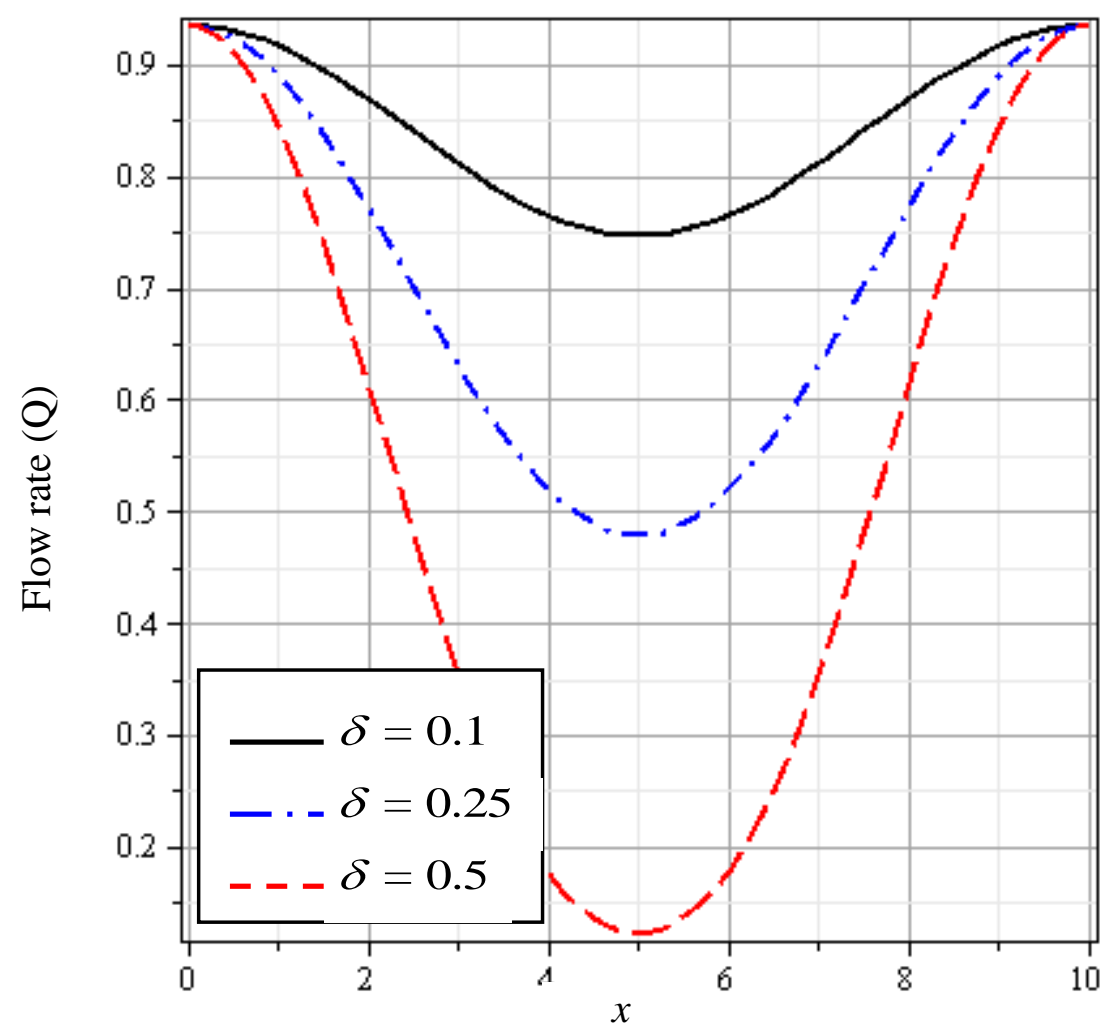

Fig.8 Variation of volumetric flow rate for different depths of the stenosis when $\operatorname{Re}=1.0, M=2.0, \quad \delta=0.25$, and $\lambda=0.30$ 


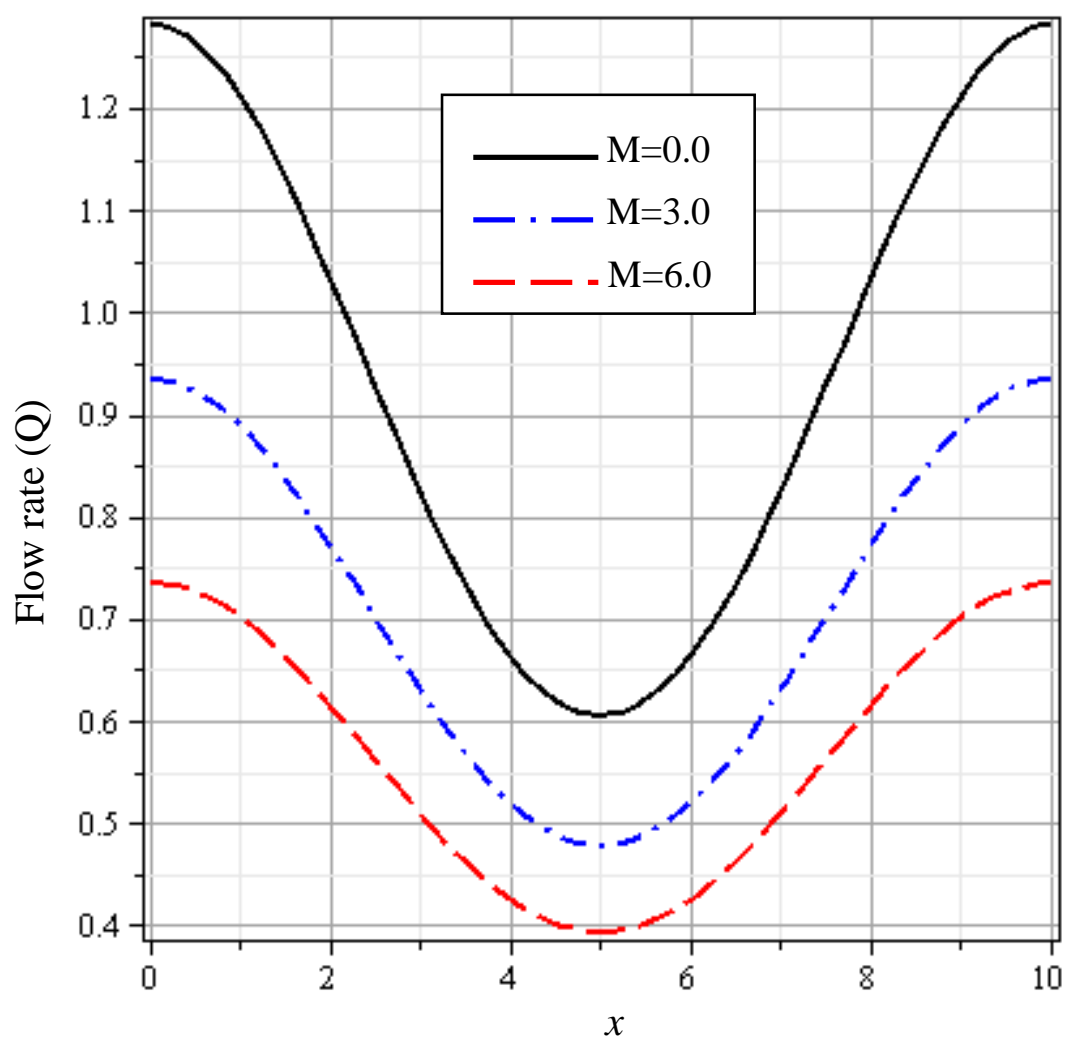

Fig.9 Variation of volumetric flow rate for different values of magnetic parameter $M$ when $\operatorname{Re}=1.0, K n=0.01 \delta=0.25$, and $\lambda=0.30$

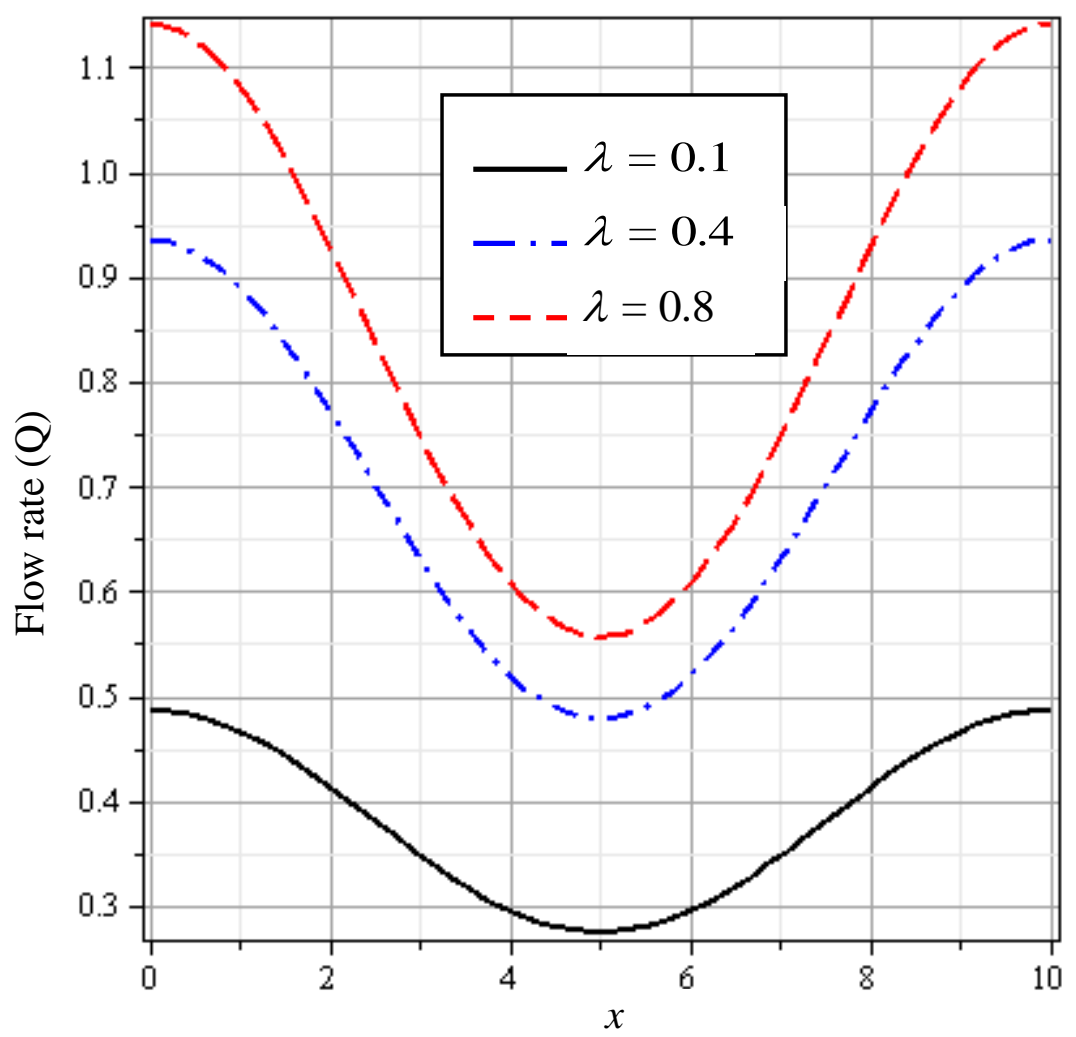

Fig.10 Variation of volumetric flow rate for different values of Darcian linear drag parameter $\lambda$ when $\operatorname{Re}=1.0, K n=0.01 \delta=0.25$, and $M=2.0$ 


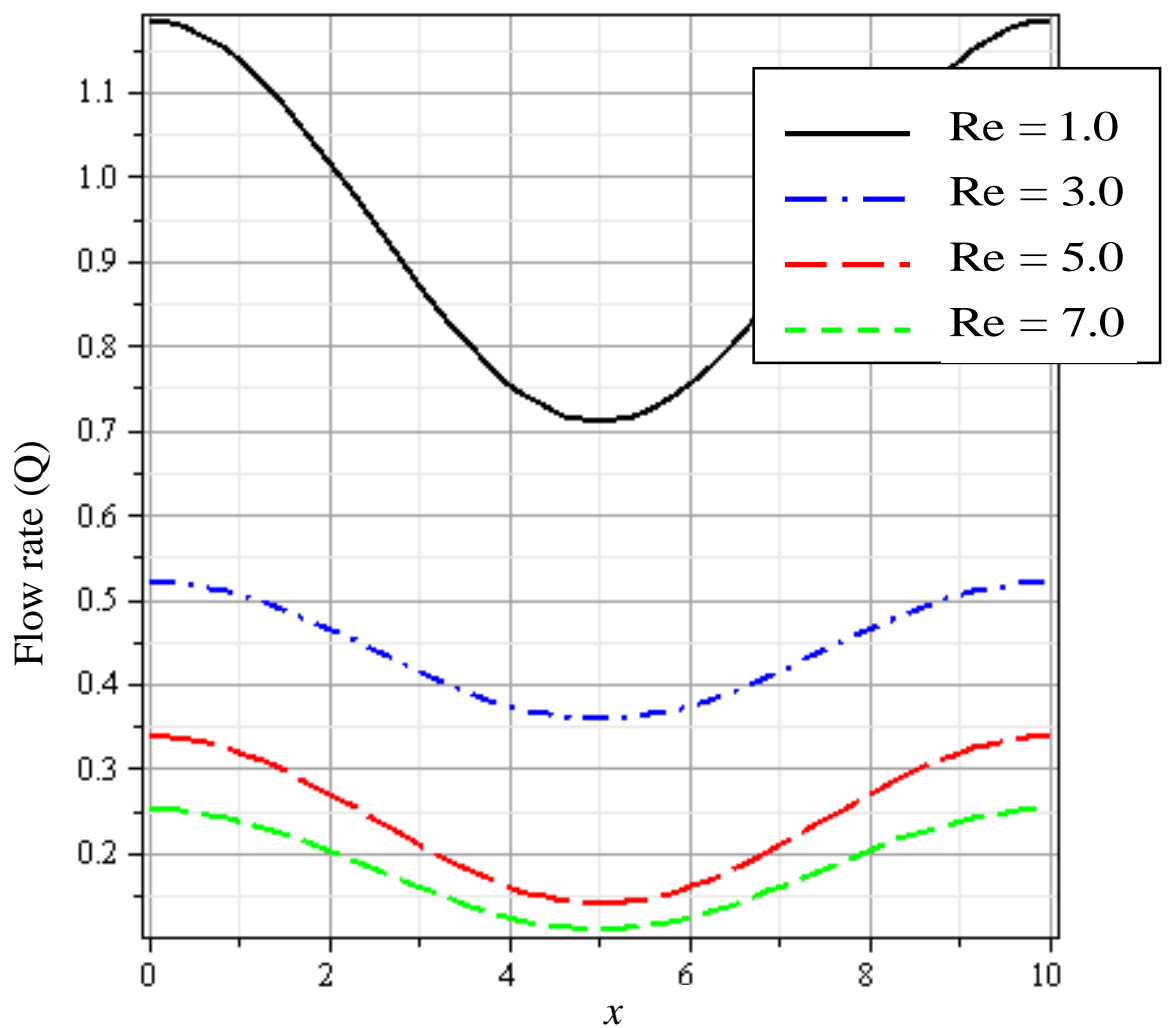

Fig.11 Variation of volumetric flow rate for different values of Reynolds number Re when $M=2.0 \mathrm{Kn}=0.01 \quad \delta=0.25$, and $\lambda=0.30$

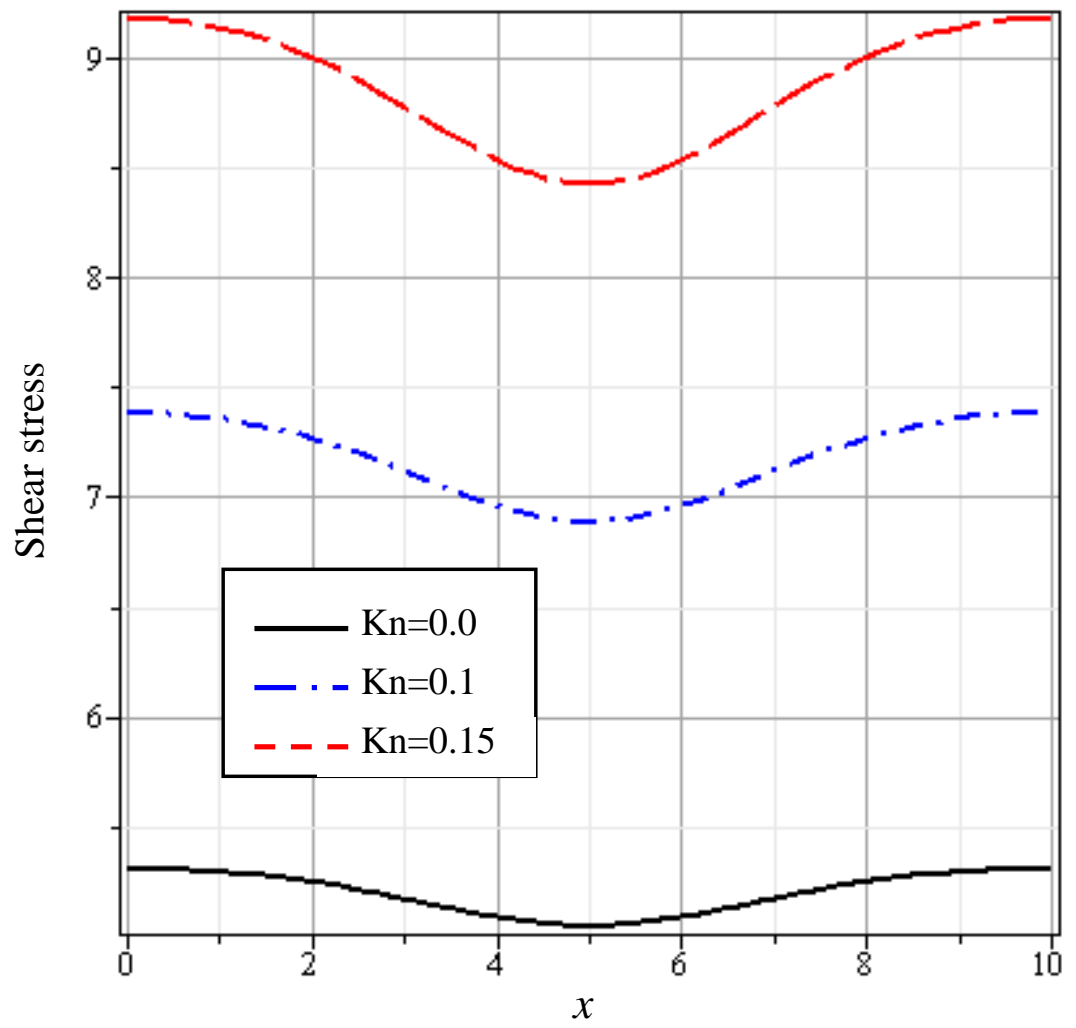

Fig.12 Variation of wall shear stress for different values of Knudsen number $K n$ when $M=2.0 \operatorname{Re}=1.0 \delta=0.25$, and $\lambda=0.30$ 
It is a widely accepted fact that the wall shear stress plays an important role in the development of the arterial diseases. Hence it is important to study the effects of the physical parameters on the wall shear stress. Fig. 12 gives the variation of wall shear stress for different values of the slip parameter (Knudsen number Kn). It may be observed that the wall shear stress increases with increasing the slip parameter. On the otherwise, the wall shear stress decreases with increasing the height of the stenosis $\delta$ as shown in Fig. 13.

Fig. 14 gives the variation of the wall shear stress for different values of the magnetic parameter M. It may observed that the wall shear stress decreases with increasing the magnetic parameter. On the otherwise, as shown in Fig. 15, the wall shear stress increases with increasing the values of Darcian linear drag parameter $\lambda$. Fig. 16 depicts the variation of the wall shear stress for different values of Reynolds number Re. it is worthwhile to note that the wall shear stress decreases as the Reynolds number Re increases. It also noted that, at strong injection the wall shear stress seems to be rectilinear.

Fig. 17 indicates the variation of the pressure gradient $(-\partial p / \partial y)$ versus time at different values of Darcian linear drag parameter $\lambda$. It is observed that the pressure gradient and its effect decreases with increases the Darcian linear drag parameter $\lambda$.

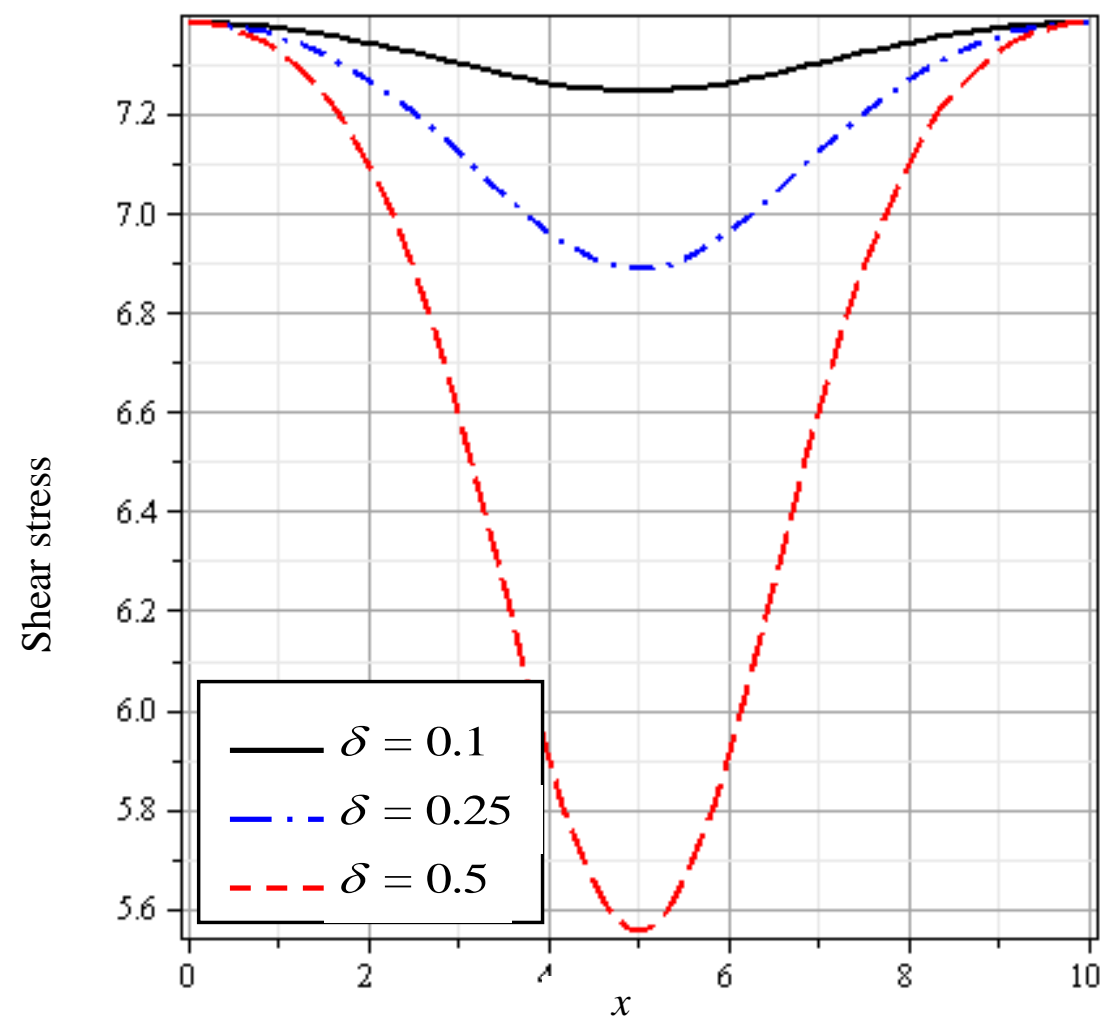

Fig.13 Variation of wall shear stress for different values of depths of the stenosis $\delta$ when $M=2.0 \operatorname{Re}=1.0 \mathrm{Kn}=0.01$ and $\lambda=0.30$ 


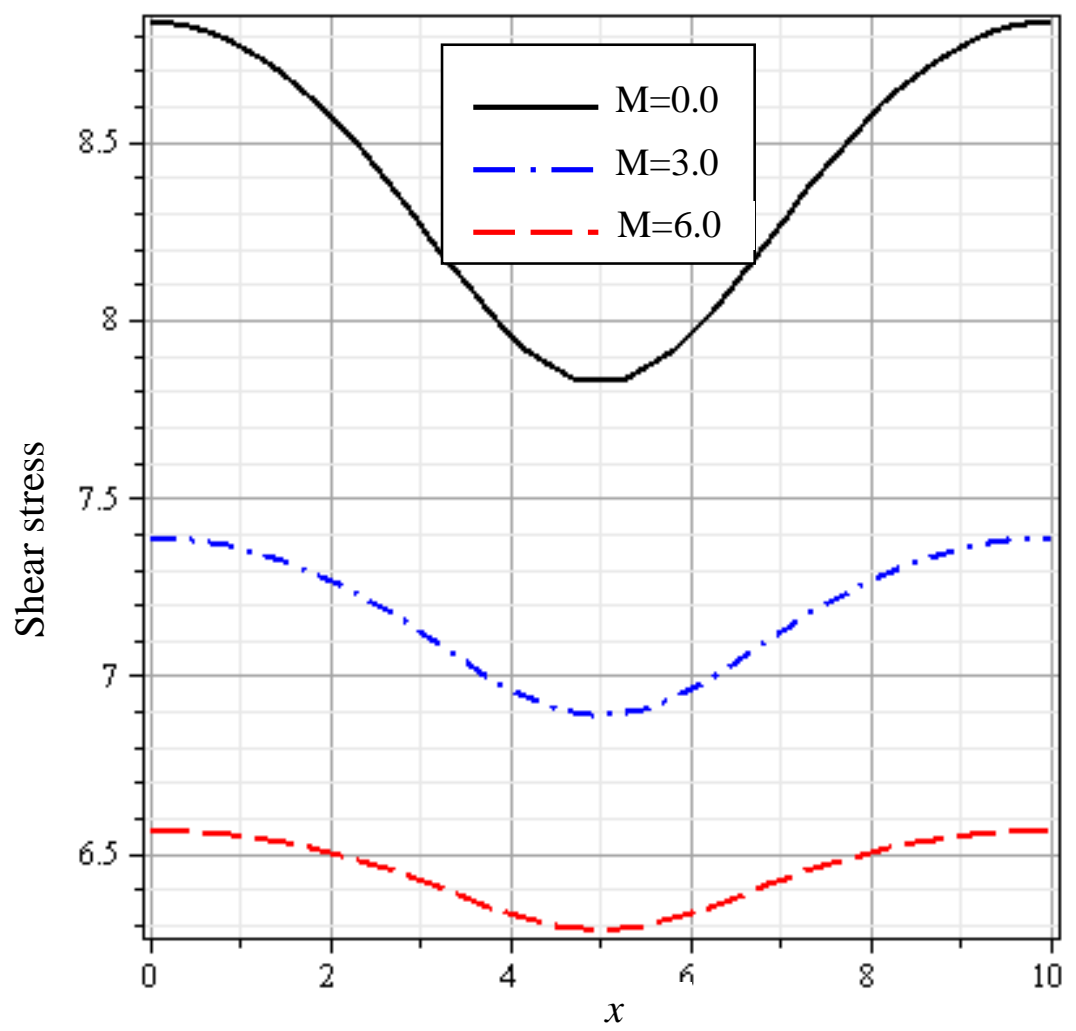

Fig.14 Variation of wall shear stress for different values of magnetic parameter $M$ when $\delta=0.25 \mathrm{Re}=1.0 \mathrm{Kn}=0.01$ and $\lambda=0.30$

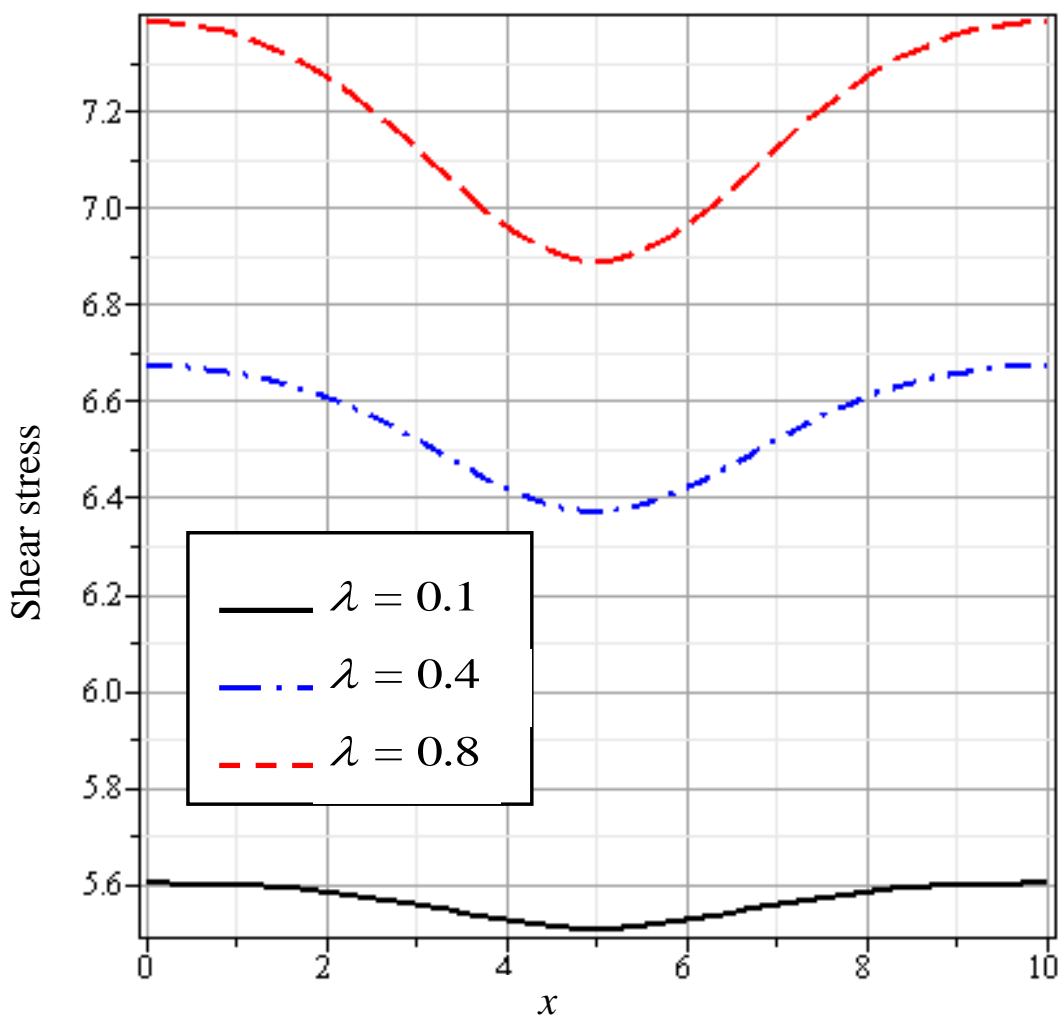

Fig.15 Variation of wall shear stress for different values of Darcian linear drag parameter $\lambda$ when $M=2.0 \operatorname{Re}=1.0 K n=0.01$ and $\delta=0.25$ 


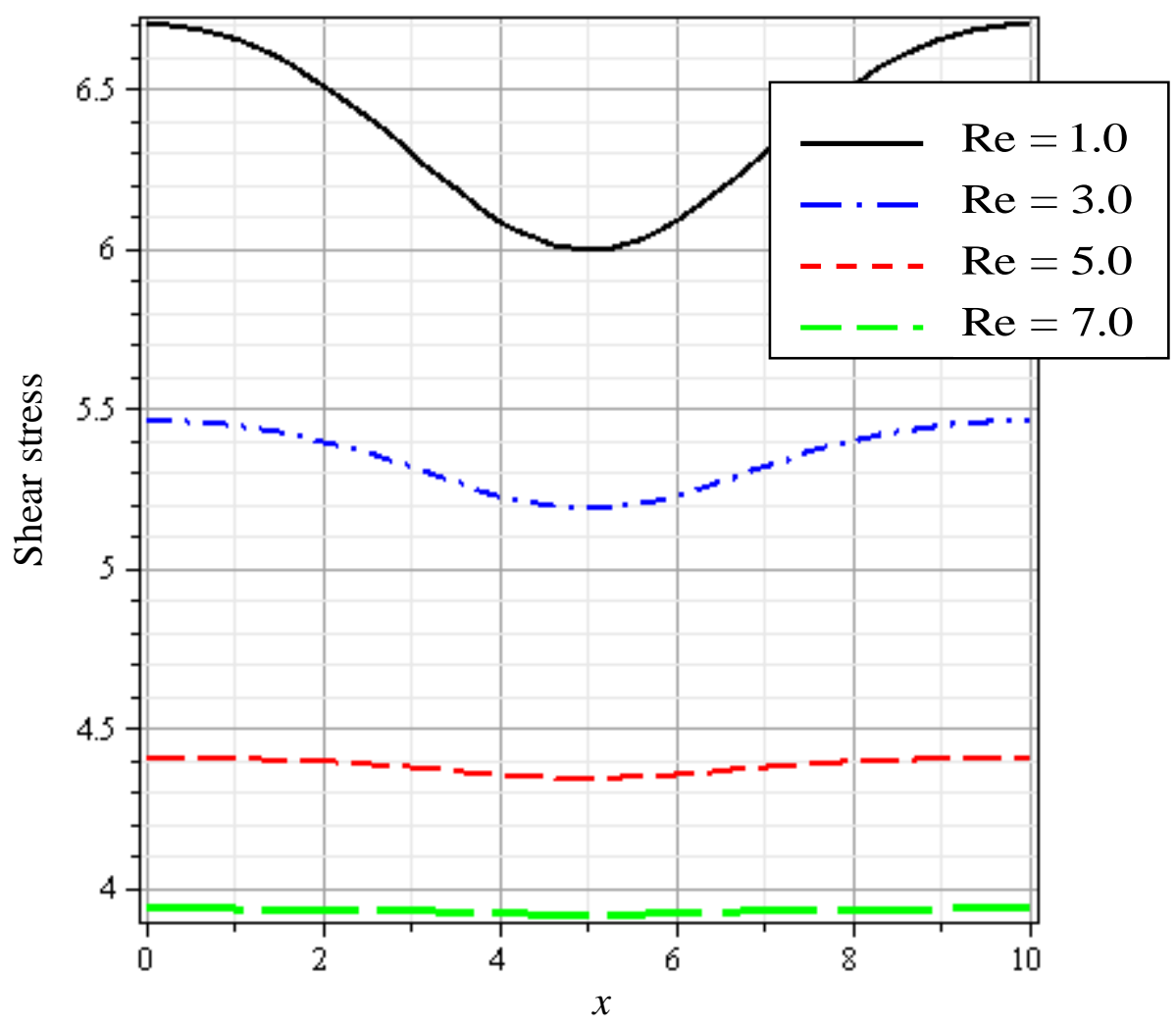

Fig.16 Variation of wall shear stress for ditterent values of Reynolds number Re when $M=2.0 \delta=0.25 \mathrm{Kn}=0.01$ and $\lambda=0.30$

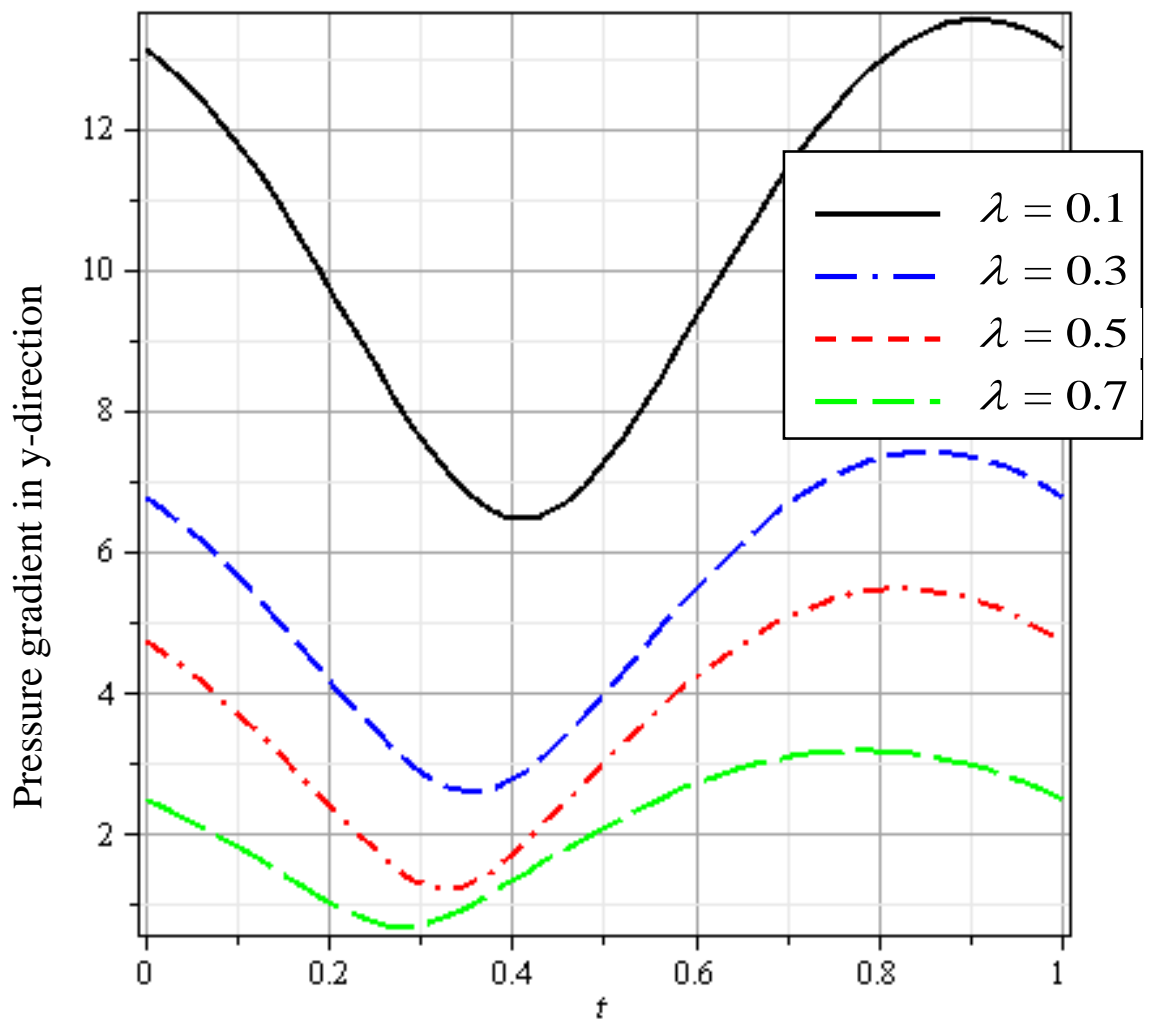

Fig.17 Variation of pressure gradient $-\partial p / \partial y$ for different values of Darcian linear drag parameter $\lambda$ when $\varepsilon=0.3$ 


\section{Conclusions}

The present study deals with a theoretical investigation of unsteady pulsatile blood flow through porous medium in a constricted porous channel subjected to time dependent suction/injection at the walls of the channel considering blood as an incompressible electrically conducting fluid. Also, to study the combined effect of magnetic field and permeable walls slip velocity. The prime concern in our present study has been to assess the role of velocity slip in blood flow through arteries and to determine those regions where the velocity is low and also the regions where the wall shear stress is low. Thus the study bears the potential to further explore the causes and development of arterial diseases like atherosclerosis and atheroma.

Consideration of velocity-slip at the permeable wall has been of prime concern in the study. From the computational results, it may be concluded that:

- The pulsatile axial velocity $u$ of the blood at the throat of the stenosis decreases with increasing the slip parameter $\mathrm{Kn}$, depth of the stenosis $\delta$, magnetic parameter $\mathrm{M}$ and Reynolds number Re (effect of injection or suction) while increases with increasing the Darcian linear drag parameter $\lambda$.

- The volumetric flow rate $\mathrm{Q}$ of the blood along the longitudinal $\mathrm{x}$-axis decreases with increasing the slip parameter $\mathrm{Kn}$, depth of the stenosis $\delta$, magnetic parameter $\mathrm{M}$ and Reynolds number Re (effect of injection or suction) while increases with increasing the Darcian linear drag parameter $\lambda$.

- It is a widely accepted fact that the wall shear stress plays an important role in the development of the arterial diseases. The shear stress $\tau_{w}$ at the permeable wall increases with increasing the slip parameter Kn and the Darcian linear drag parameter $\lambda$ while decreases with increasing the depth of the stenosis $\delta$, magnetic parameter M and Reynolds number Re (effect of injection or suction).

- The pressure gradient decreases with increases the Darcian linear drag parameter $\lambda$. Also, the pressure gradient in $y$-direction is affected only by time and Darcian linear drag parameter $\lambda$.

\section{References}

\section{References}

[1] E. F. Elshehawey and S. Z. A. Husseny, 2002, Peristaltic transport of a magnetofluid with porous bounderies, Applied Mathematics and Computation, $129 \mathrm{pp}$ 421-440.

[2] A. Sinha and J. C. Misra, 2012, Influence of slip velocity on the blood flow through an artery with permeable wall: a theortical study, Int. J. of Biomathematics, 5(5) pp 1250042-1-20.

[3] O. D. Makinde and E. Osalusi, 2006, MHD steady flow in a channel with slip at the permeable boundaries, Romanian Journal of Physics, 51 pp 319-328. 
[4] K. Elangovan and N. P. Ratchaga, 2010, Steady flow through a circular vertical pipe with slip at the permeable boundaries with an applied magnetic field, Applied Mathematical Sciences, 4(50) pp 2445-2452.

[5] O. D. Makinde and T. Chinyoka, 2001, Unsteady MHD flow in a porous channel with an exponentially decreasing suction, Journal of Pure and Applied Mathematics, 1(1) pp 1-13.

[6] A. Sattar and A. Waheedullah, 2013, Unsteady flow of a visco-elastic fluid through porous medium bounded by two porous plates, International Journal of Engineering Sciences and Technology, 5(2) pp 329-334.

[7] X. Si, L. C. Zheng, X. X. Zheng and Y. Chao, 2011, Homotopy analysis solutions for the asymmetric laminar flow in a porous channel with expanding or contracting walls, Acta Mech. Sin., 27(2) pp. 208-214.

[8] I. M. Eldesoky, 2012, Slip effects on the unsteady MHD Pulsatile blood flow through porous medium in an artery under the effect of body acceleration, International Journal of Mathematics and Mathematical Sciences, Vol. 2012 pp 860239-1-26.

[9] G. S. Beavers and D. D. Joseph, 1967, Boundary conditions at a naturally permeable wall, Journal of Fluid Mechanics, 30(1) pp 197-207.

[10] E. F. El-Shehawey, N. T. El-Dabe, and I. M. El-Desoky, 2006, Slip effects on the peristaltic flow of a non-Newtonian Maxwellian fluid, Journal of Acta Mechanica 186 pp 141 - 159.

[11] I. M. Eldesoky, 2012, influence of slip condition on peristaltic transport of a compressible Maxwell fluid through porous medium in a tube, International Journal of Applied Mathematics and Mechanics, 8(2) pp 99 - 117.

[12] W. Kwang and J. Fang, 2000, Peristaltic transport in a slip flow, European Physical Journal B, 16 pp 543-547.

[13] I. M. Eldesoky, 2013, Effect of relaxation time on MHD pulsatile flow of blood through porous medium in an artery under the effect of periodic body acceleration, Journal of Biological Systems, 21(2) pp 135001-1-17.

[14] Y. C. Wang, 1971, Pulsatile flow in a porous channel, Journal of Applied Mechanics, 38 pp 533-553.

[15] S. Tsangaris, D. Kondaxakis, and N. W. Vlachakis, 2007, Exact solution for flow in a porous pipe with unsteady wall suction/injection, Journal of communications in nonlinear science and numerical simulation 12, pp. 11811189.

[16] J. V. Ramanmurthy, N. Srinivasacharyulu, and O. Odelu, 2007, Viscous fluid flow between two parallel plates with periodic suction and injection. A.M.S.E journal on Modeling, Series B: Mechanics and Thermics, 50 (2) pp. 29 - 37.

[17] J. V. R. Murthy and N. K. Bahali, 2009, Steady flow of micropolar fluid through a circular pipe under a transverse magnetic field with constant suction/injection, International Journal of Applied Mathematics and Mechanics, 5(3) pp. 1-10.

[18] D. Srinivasacharya, N. Srinivasacharyulu and O. Odelu, 2011, Flow of couple stress fluid between two parallel porous plates, International Journal of Applied Mathematics, 41(2) pp. 10-14. 
[19] S. Rajender, S. Sreennadh, Y. V. K. Kumar and S. V. H. Kumari, 2012, Pulsatile flow of a couple stress fluid in a channel bounded by permeable beds with suction and injection, 3(1) pp. 36-45.

[20] S. Kumar and C. Diwakar, 2012, A biomagnetic fluid dynamic model for the MHD Couette flow between two infinite horizontal parallel porous plates, Journal of Ultra Scientist 24(3) pp. 483-488.

[21] J. Singh and R. Rathee, 2010, Analytical solution of two-dimensional model of blood flow with variable viscosity through an indented artery due to LDL effect in the presence of magnetic field, Int. J. of the Physical Sciences, 5(12) pp 18571868.

[22] M. Jain, G. C. Sharma and A. Singh, 2009, Mathematical analysis of MHD flow of blood in very narrow capillaries, Int. J. E. Transactions B: Applications, 22(3) pp 307-315.

[23] R. Mettal, S. P. Simmons and F. Najjar, 2003, Numerical study of pulsatile flow in a constricted channel, Journal of Fluid Mechanics, 485 pp. 337-378.

[24] Y. Abdelmaboud and Kh. S. Mekheimer, 2012, Unsteady pulsatile flow through a vertical constricted annulus with heat transfer, Naturforschung A, Z Naturforsch. 67a, 185-194.

[25] Kh. S. Mekheimer and M. A. Elkot, 2008, Magnetic field and Hall currents influences on blood flow through a stenotic artery, Journal of Applied Mathematics and Mechanics, 29 (8) pp 1-12.

[26] Kh. S. Mekheimer, M. H. Haroun and M. A. Elkot, 2011, Induced magnetic field influences on blood flow through an anisotropically tapered elastic arteries with overlapping stenosis in an annulus, Candian Journal of Physics, 89 (2) pp 201212.

[27] N. Verma and R. S. Parihar, 2009, Effects of Magneto-hydrodynamic and hematocrit on blood flow in an artery with multiple mild stenosis, International Journal of Applied Mathematics and Computation, 1(1) pp 30-46.

[28] P. Nagarani and G. Sarojamma, 2008, Effect of body acceleration on pulsatile flow of Casson fluid through a mild stenosed artery, Korea-Australia Rheology Journal, 20(4) pp 189-196.

[29] B. K. Mishra and N. Verma, 2007, Effect of porous parameter and stenosis on the wall shear stress for the flow of blood in human body, Research Journal of Medicine and Medical Sciences, 2(2) pp 98-101.

[30] A. Sinha, J. C. Misra and G. C. Shit, 2011, Mathematical modeling of blood flow in porous vessel having double stenosis in the presence of an external magnetic field, International Journal of Biomathematics, 4(2) pp 207-225.

[31] S. Mishra, S. U. Siddiqui and A. Medhavi, 2011, Blood flow through a composite stenosis in an artery with permeable wall, Applications and Applied Mathematics: An International Journal AAM, 6(1) pp. 58-73.

[32] G. C. Shit and M. Roy, 2012, Hydromagnetic pulsating flow of blood in a constricted porous channel: A theoretical study, Proceedings of the World Congress on Engineering, Vol I, July 4-6, London, U. K.

[33] P. G. Saffman, 1971, On the boundary conditions at the surface of a porous medium, Stud. Appl. Math. 50 pp. 93. 
[34] B. S. Bhatt and N. C. Sacheti, On the analogy in slip flows, Indian J. Pure Appl. Math., 10(3) pp.303-306. 